\title{
Evaluate the Efficacy of Novel Nursing Management Method Including Both Preparation Services and Continuity of Care in Elderly Patients with Aspiration Pneumonia
}

\author{
Xiulan Deng ${ }^{1, \dagger}$, Yan Zhou ${ }^{2, \dagger}$, Yanli Yang ${ }^{1}$, Nijun Jiang ${ }^{1}$, Xizhi Zhang ${ }^{1}$, Ruiying Huang ${ }^{4}$, \\ Shanying Zhang ${ }^{1}$, Xingdong Cai ${ }^{1}$, Jiewei Huang ${ }^{3}$, * \\ ${ }^{1}$ Department of Respiratory, the First Affiliated Hospital of Jinan University, Guangzhou, China \\ ${ }^{2}$ Department of Endocrinology, the First Affiliated Hospital of Jinan University, Guangzhou, China \\ ${ }^{3}$ Nursing Department, the First Affiliated Hospital of Jinan University, Guangzhou, China \\ ${ }^{4}$ Extended Care Clinic, the First Affiliated Hospital of Jinan University, Guangzhou, China
}

Email address:

chgzhjw@sohu.com (Jiewei Huang)

*Corresponding author

$\dagger$ Xiulan Deng and Yan Zhou are co-first authors.

\section{To cite this article:}

Xiulan Deng, Yan Zhou, Yanli Yang, Nijun Jiang, Xizhi Zhang, Ruiying Huang, Shanying Zhang, Xingdong Cai, Jiewei Huang. Evaluate the Efficacy of Novel Nursing Management Method Including Both Preparation Services and Continuity of Care in Elderly Patients with Aspiration Pneumonia. American Journal of Nursing Science. Vol. 10, No. 1, 2021, pp. 36-40. doi: 10.11648/j.ajns.20211001.17

Received: December 22, 2020; Accepted: January 7, 2021; Published: January 22, 2021

\begin{abstract}
Objective: The concept of discharge preparation services originated from the continuum of care and referral system first established in the United States in 1910. Extended care is a series of nursing care activities to ensure continuity and coordination of care for patients in different health care settings. Guidance to reduce or prevent the deterioration of the health status of patients with chronic diseases. The aim of our study was to evaluate the efficacy of discharge preparation service and extended care in elderly patients with aspiration pneumonia. Methods: A total of 40 elderly patients with aspiration pneumonia who were hospitalized in the respiratory and critical care department of a tertiary care hospital from February 2017 to February 2019 were enrolled in this study and divided into two groups. Patients in the control group received conventional nursing interventions while patients in observation group received specific chronic disease management including discharge preparation service and continuity of care. Patients' satisfaction with nursing services, their ability to take care of themselves in daily life one month after discharge, and the number of hospital readmissions within one year after discharge were documented and compared between two groups. Results: Our study suggested that patients' satisfaction with nursing services and their self-care capabilities one month after discharge were significantly higher in the observation group compared to the control group $(\mathrm{P}<0.05)$. Meanwhile, the number of readmissions within one year after discharge was significantly decreased in the observation group than in the control group $(\mathrm{P}<0.05)$. Conclusion: The chronic disease management model including specific discharge preparation service and extended care provided patients with better nursing services and resulted in better prognosis.
\end{abstract}

Keywords: Discharge Preparation Service, Extended Care, Elderly Aspiration Pneumonia, Chronic Disease Management Model

\section{Introduction}

The concept of discharge preparation services originated from the continuum of care and referral system first established in the United States in 1910. It refers to a specific health care team that provides appropriate care to patients from the time they are admitted to the hospital and integrates the required health resources according to surrounding environment and situation to ensure the patient can be discharged from the hospital without unpleased events [1]. Studies have shown that effective discharge preparation can reduce the incidence of post-discharge complications and 
readmission rates, thereby saving medical resources and reducing the burden on families [2]. Extended care is a series of nursing care activities to ensure continuity and coordination of care for patients in different health care settings. Guidance to reduce or prevent the deterioration of the health status of patients with chronic diseases [3]. Age, underlying disease, impaired consciousness, swallowing disorders and nasal feeding are common causes of aspiration pneumonia in elderly patients [4-6] that can lead to death in severe cases [7, 8]. To better address the risk factors mentioned above, the Department of Respiratory and Critical Care Medicine of our hospital developed a specific nursing care management based on the successful experience of Taiwan's discharge preparation service that including both discharge preparation service and extended care to elderly patients. In this study, the efficacy of this novel manage model was evaluated in patients with aspiration pneumonia.

\section{Data and Methods}

\subsection{Research Objects}

A total of forty elderly patients with aspiration pneumonia who were hospitalized in the Department of Respiratory and Critical Care Medicine of a tertiary hospital in Guangzhou from February 2017 to February 2019 were enrolled for the study. Inclusion criteria: (1) patients with a clear diagnose of aspiration pneumonia and presented with dysphagia, nasal feeding, impaired consciousness and other related risk factors, new pulmonary infiltrative lesions on chest X-ray, age over 60 years old. (2) Need special extended care services (1) low self-care ability: ADL score $<40$, incapacitated, dementia patients. (2) long-term home oxygen therapy, ventilator ventilation, tracheotomy care. (3) swallowing function training, pulmonary rehabilitation training, limb movement rehabilitation training. (4) Retention of various tubes: such as gastric tube, nasogastric tube, ureter, PICC, infusion port, gastrostomy tube. (5) Wound/stoma: pressure ulcers, surgical wounds, diabetic foot, bladder fistula, artificial anus. (6) Repeated hospitalization within a short period of time. (3) Patient or family consent for referral to Continuing Care Services.

Exclusion criteria: critically or terminated ill, with severe cardiac, cerebral or renal diseases.

All patients were divided into two groups. A total of 18 males and 5 females were assigned to the control group, with an age range of 62-90 years and an average age of $75.26 \pm 9.88$ years. A total of 14 males and 3 females were assigned to the observation group, with an age range of 70-95 years and an average age of $82.00 \pm 6.40$ years. The baseline characteristics between the two groups were comparable with no statistically significant difference $(\mathrm{p}>0.05)$.

\subsection{Research Methods}

\subsubsection{Team Formation}

The Department of Respiratory and Critical Care Medicine and Continuing Care Services formed a Discharge Preparation Services team, consisting of the Respiratory and Critical Care
Medicine director, head nurse, specialty nurses, and Continuing Care Services nurses. All team members received proper training before the onset of this study.

\subsubsection{Research Methods}

\section{i. Control Group}

Patients in the control group received standard care including the provision of specialized care for patients through assessment during hospitalization, health education for patients and their families, discharge instructions, dietary and medication instructions, and regular visits by extended care staff to replace gastric tubes and other tubes according to patients' needs.

\section{ii. Observation Group}

In addition to the control group, a chronic disease management model of discharge preparation services and continuity of care were also provided to the patients in the observation group, as introduced below.

\section{iii. Hospital Discharge Preparation Service}

(1) Patients screening On the second day of admission, the responsible nurse evaluated the patient according to the referral criteria.

(2) Assessment of Patients Patients requiring special care were further evaluated and intervened by the nurse specialist on the third day of admission, including: (1) Patient self-care assessment, muscle strength assessment, cough and sputum assessment, swallowing assessment, psychological assessment, nutritional assessment, deep vein thrombosis risk assessment, fall risk factor assessment, NORTON assessment, etc.; (2) From family aspects: The type of care required; psychological concerns of family and caregivers, and family expectations; (3) Medical equipment needed: e.g. suction machine, blender, home oxygen machine, air mattress, etc. Other assessments: family financial status, social support, etc.

(3) Discharging plan

Through assessment, the nurse specialist identified the most important problems and nursing skills that were needed for follow-up care after the patient was discharged from the hospital and prepared a preliminary discharge plan including: (1) Operational skills instruction: for family/caregiver aspects including nasal feeding, suctioning, back patting, sputum drainage and passive patient movements. For patients: coughing and coughing sputum method, physical exercise methods (ankle pump, pulling up and sitting on bed rail, stretching, abduction and flexion movements, etc.), swallowing rehabilitation training skills, turning over skills, etc. (2) knowledge guidance: gastric emptying test before nasal feeding, nasal feeding volume, temperature, interval, food configuration and storage as well as position and gastric tube fixation method. (3) Home medical equipment instruction: such as home oxygenator, sputum aspirator use and daily maintenance. (4) Instruction on emergencies such as if the gastric tube was accidentally detached, seek immediate help and do not feed by mouth without swallowing assessment.

(4) Referral for Continuing Care (1) The nurse specialist explained the necessity of continuing care to patient's family 
and provided necessary instructions. (2) The nurse specialist communicated with the physician in charge to discuss the patient's follow-up care needs after discharge. (3) The nurse specialist discussed with the physician in charge, the charge nurse, the nurse from the Continuing Care Services Department, and the patient/family to develop a final discharge plan and finalized the discharge plan.

(5) Implementing the discharge plan The responsible nurse provided proper care and instructions following patient's specific discharge plan in a step-by-step manner twice a day (once in the morning and once in the afternoon). The responsible nurse would guide the patient and/or family member/caregiver until the process had been mastered.

(6) Assessment of required skills A nurse would evaluate the skills and knowledge of the patient, family members and caregivers that required for the continuing care before patient discharge.

\section{iv. Extended Care}

The Extended Care Service Department was responsible for developing individual follow-up plan after patient's discharge that could be carried out in three ways: telephone follow-up, in-home follow-up, and WeChat follow-up.

(1) Telephone follow-up Nurses from the Extended Care Service Department conducted the first telephone follow-up visit within 3 Days after patient discharge. The main contents of the telephone follow-up visit were consisted of general condition of the patient, the condition of the indwelling tubes, the sputum volume, the coughing and coughing sputum, and the eating condition, etc.

(2) Home visits The first home visit was usually conducted within one month after patient discharge and then based on the patient's health conditions and/or the needs of the family/caregiver. The information collected included: (1) Patient assessment: vital signs, self-care ability, general skin condition, effective coughing and sputum management, swallowing function, motor skills, psychological assessment, compliance assessment (including medications and rehabilitation exercises), etc. (2) Family members or caregivers were asked to provide a comprehensive assessment of the patient's health containing acquisition of various operational skills, assessment of family caregiving strength and their psychological assessment. (3) Home environment assessment. (4) Skill instruction: problems identified during the assessment should be corrected and re-instructed; for patients unable to get out of bed, bed exercises (ankle pump, bicycle in bed, etc.) should be instructed; patients with lower limb muscle strength level 3 or above would be instructed on how to perform walking exercises with the help from family caregiver; for patients with poor compliance, family caregiver would be instructed to supervise and instruct. If the home environment was not conducive to patient's recovery and activities or there were potential safety hazards, the family would be advised to modify the home environment.

(3) WeChat follow-up WeChat follow-up, as a new mode of follow-up, is more intuitive and convenient in collecting patients' information and health conditions through WeChat text, voice, pictures, videos, etc.

The Barthel Index was used to evaluate the patients' daily behavioral ability, which included 10 items: eating, dressing, bathing, grooming, controlling urination and defecation, toileting, transferring from bed to chair, walking on level ground, and going up and down stairs. The total score was 100 points. Patients with a total score of $\leq 40$ points was characterized as heavy dependence, (41-60) for moderate dependence, (61-99) for light dependence, and 100 points for no dependence. (ii) The number of readmissions within 1 year after patient's discharge was documented. (3) To evaluate patients' satisfaction with nursing services before discharge, nursing department of the hospital developed a nursing satisfaction questionnaire for inpatients, which includes 5 aspects: service quality, education and awareness, communication, explanation, service process, and environment and facilities with a total of 10 items and each item has 4 options, namely very satisfied, satisfied, average and dissatisfied, corresponding to a score of 10, 8, 5, 0 .

\subsubsection{Statistical Analysis}

Statistical analysis was performed using the spss 13.0 software, and the measures were described by $\mathrm{x} \pm \mathrm{s}$, with $P<0.05$ considered as the statistically significant difference.

\section{Result}

\subsection{Comparison of Daily Life Self-care ability (Barthel Index) Between Two Groups of Patients at Admission and One Month After Discharge (Tables 1 and 2)}

At the time of admission, there was no significant difference in the ability of daily living between the control group and the observation group $(P=0.975)$; one month after discharge, the ability of daily living in the observation group was significantly better than that in the control group $(P=$ 0.011).

Table 1. Patients' self-care ability (Barthel index) at the time of hospitalization.

\begin{tabular}{llllllll}
\hline Group & Case & No depend & Light depend & Moderate depend & Severe depend & \multicolumn{1}{c}{$\boldsymbol{P}$} \\
\hline Control & 23 & 0 & 1 & 4 & 18 & 0.05 \\
Observe & 17 & 0 & 1 & 3 & 13 & \\
\hline
\end{tabular}

Table 2. Patients' self-care ability (Barthel index) at the time a month of discharge.

\begin{tabular}{lcllllll}
\hline Group & Case & No depend & Light depend & Moderate depend & Severe depend & $\boldsymbol{\chi}$ & \multicolumn{2}{c}{} \\
\hline control & 23 & 1 & $\mathbf{4}$ & 6 & 12 & 11.123 \\
Observe & 17 & 2 & $\mathbf{5}$ & 3 & 7 & 0.011 \\
\hline
\end{tabular}




\subsection{Number of Readmissions Within one Year After Patient Discharge}

Compared to the control group, the number of readmissions at 1 year after discharge was significantly reduced in the observation group $\left(X^{2}=7.047, p=0.029\right)$, as shown in Table 3 .

Table 3. Comparison of the number of readmissions within 1 year after patient discharge between two groups.

\begin{tabular}{lclllcl}
\hline group & case & 0time & 1 time & 2times & $\boldsymbol{\chi 2}^{\mathbf{2}}$ & $\boldsymbol{P}$ \\
\hline control & 23 & 7 & 10 & 6 & 7.047 & 0.029 \\
observe & 17 & 12 & 4 & 1 & & \\
\hline
\end{tabular}

\subsection{Patient Satisfaction with Nursing Services Before Hospital Discharge}

Our data suggested that patients in the observation group was significantly more satisfied with nursing services than patients in the control group $(t=4.067, p=0.0002)$, as shown in Table 4.

Table 4. Comparison of nursing service satisfaction between two groups before hospital discharge.

\begin{tabular}{lll}
\hline group & case & service satisfaction \\
\hline control & 23 & $87.3 \pm 0.8165$ \\
observe & 17 & $92.06 \pm 0.4886$ \\
$t$ & & 4.067 \\
$P$ & & 0.0002 \\
\hline
\end{tabular}

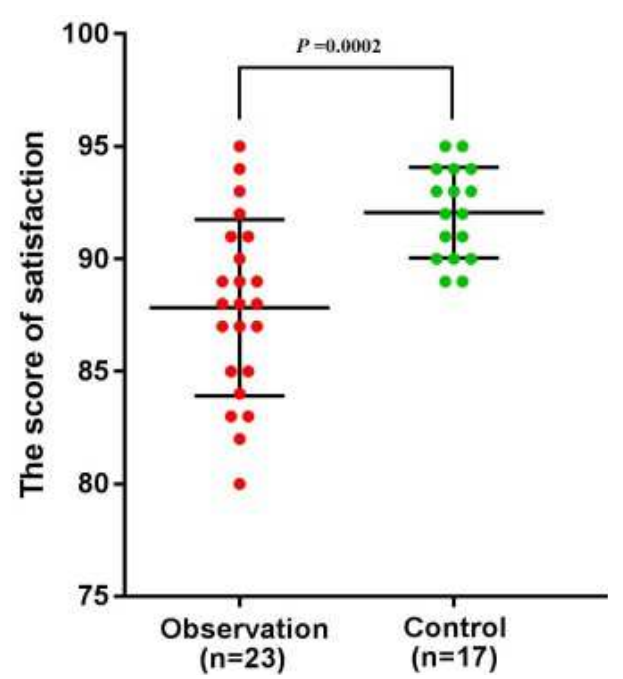

Figure 1. Comparison of nursing service satisfaction between two groups before hospital discharge.

\section{Discussion and Conclusion}

Discharge preparation service provides patients with complete and continuous care during transformation of care environment, therefore reduces the incidence of complications and readmission rate after discharge, save medical resources, medical costs and reduces family burden. Continuing care refers to the continuous medical follow-up and guidance from the hospital to the family or nursing home after the patients return to their family or community so as to reduce or prevent the deterioration of the health. The chronic disease management mode between the discharge preparation service and continuing care of close cooperation and connection plays an important role in improving the self-care ability of patients, reducing the number of re-hospitalization and improving the satisfaction of patients with nursing service.

Risk factors for developing aspiration pneumonia in elderly patients such as advanced age, underlying disease, impaired consciousness, and nasal feeding are commonly associated with the decline in patients' self-care capability. In this study, we provided patients with disease related knowledge and nursing skills through a chronic disease management model linking discharge preparation service and extended care as well as guided patients to perform rehabilitation exercises which resulted in a lower re-admission rate. The present study showed that the self-care ability of patients in the intervention group was significantly higher than that of the control group ( $p$ $<0.05$ ). This discovery was consistent with another study [9] that also showed that the implementation of discharge preparation services and home care for stroke patients improved the patients' self-care ability.

At present, the main diagnosis and treatment of patients with chronic diseases is still hospital-based, which to some extent increases the burden on patients' families and hospitals. Elderly patients with aspiration pneumonia are commonly complicated with multiple chronic diseases other than lung diseases. Furthermore, immobility is not uncommon among these patients who often required prolonged hospital stays due to the need for nasal feeding and airway care. It has been known that for such patients, the incidence of disease recurrence was relatively high [10]. The conventional nursing care model only provides limited discharge guidance when patients are discharged from the hospital. It does not provide targeted and personalized guidance to patients and their families, and does not assess their actual ability to conduct the necessary care to the patient. Unfortunately, this traditional care is often unsuccessful and might contribute to repeated hospitalization. Therefore, the Department of Respiratory Medicine of our hospital combined the chronic disease management model and discharge preparation service and developed a novel nursery management for elderly patients with aspiration pneumonia. This novel management enables patients to receive timely extended care service and continuous home services after hospital discharge. The results of this study showed that the number of hospital readmissions in the observation group was significantly decreased within one year after discharge compared to the control group and was consistent with the findings of Henke RM [11] and others. Furthermore, Chen Qin [12] and Liang Yuping [13] demonstrated that discharge preparation and extended care for patients with COPD reduced the number of readmissions.

More and more evidences suggested that nurse-led discharge preparation services could improve patient 
satisfaction with nursing services [14]. The results of this study showed that the nursing service satisfaction was significantly higher in the observation group than in the control group $(\mathrm{p}<0.05)$. The reason might be that during the intervention process, the nursing staff provided patients with disease-related health education and nursing skills during hospitalization, and also provided patients with telephone follow-ups, home visits and micro-phone-based follow-ups after discharge to meet the actual needs of patients. This observation suggested the importance of nursing staff and is conducive to establishing a good nurse-patient relationship with patients, thus increasing the satisfaction of patients and/or their families with nursing services. This discovery was in consistent with previous studies showing the discharge preparation services on elderly patients with chronic diseases [15] and continuity care for patients with chronic diseases [16] significantly improved patient satisfaction.

Overall, the novel chronic disease management that links discharge preparation and continuing care provided patients with a continuum of care, improved caregivers' skills and knowledge, enabled homebound patients to receive better medical care, and more importantly, a decreased readmission rate. However, more studies including larger simple size are needed to better confirm and understanding our observation.

\section{Ethics Approval and Consent to Participate}

Not applicable.

\section{Competing Interests}

The authors declare that they have no competing interests.

\section{Fund}

Guangdong Medical Science and Technology Research Fundation. Grant Number: A2020574.

\section{References}

[1] Xi shuhua, Zhao jijun, Zhao jianhua. General situation and Enlightenment of discharge preparation service in Affiliated Hospital of Chengdu University [J]. Chinese Journal of nursing. 2007, 42 (4): 341-342.

[2] Liu qin, Zhang yuan, Ruan linyun, Research status of clinical application of discharge preparation service [J] Journal of nursing education, 2016, 31 (17): 1561-1565.
[3] Boockvar K, Vladeck BC. Improving the quality of transitional care for persons complex careneeds [J]. J Am Geriatr Soc. 2004, 52 (5): $855-856$.

[4] Smith CJ, Kishore AK, Vail A, et al. Diagnosis of stroke-associated pneumonia: recommendations from the pneumonia instroke consensus group [J]. Stroke, 2015; 46 (8): $2335-40$.

[5] Yang li, Jiang yuhua, Zhang xue. Analysis of related factors and prevention of aspiration pneumonia in elderly patients $[\mathrm{J}]$ Chinese Journal of hospital infection, 2016; 26 (13): 2948-50.

[6] Bao longmei, Wang yanyan, Lv aili. Effect of swallowing feeding intervention on aspiration pulmonary infection in patients with cerebral hemorrhage $[\mathrm{J}]$ Chinese Journal of hospital infection, 2017; 27 (21): 5018-21.

[7] Vinton DT, Capp R, Rooks SP, et al. Frequent users of US emergency departments: characteristics and opportunities for intervention. Emerg Med J 2014; 31: 526-32.

[8] Lehnert T, Heider D, Leicht H, et al. Review: health care utilization and costs of elderly persons with multiple chronic conditions. Med Care Res Rev 2011; 68: 387-420.

[9] Qin suping, Gao yakun, Gao jing. Analysis of the effect of discharge preparation plan and home care for stroke patients [J] Chinese Journal of nursing, 2014, 49 (11): 1337-1342.

[10] Mandell LA, Niederman MS. Aspiration Pneumonia [J] N Engl J Med. 2019, 380 (7): 651-663.

[11] Henke RM, Karaca Z, Jackson P, et al. Discharge planning and hospital readmissions [J]. Medical Care Research \& Review Mcrr, 2017, 74 (3): 345-368.

[12] Chen qin. Evaluation of the effect of discharge planning model in patients with chronic obstructive pulmonary disease [D]. Fuzhou: Fujian Medical University, 2011.

[13] Liang yvping Liu xin. Effect of continuous nursing on quality of life and readmission rate of patients with chronic heart failure [J]. General nursing, 2017, 15 (04): 492-495.

[14] Brook N. Nurse-led discharge planning improves quality of care [J]. Nurs Times, 2001, 97 (19): 40.

[15] Gai qiongyan, Wu mengting, Li cheng. Systematic evaluation of intervention effect of discharge preparation service on elderly patients with chronic diseases [J]. Chinese Journal of evidence based medicine, 2020, 20 (03): 289-295.

[16] Jin yi, Shi yan, Gong meifang, Sun xiao. Implementation and effect of continuous nursing model centered on patients with chronic diseases [J]. Chinese Journal of nursing, 2015, 50 (11): 1388-1391. 